\title{
ARTICLES
}

\section{TRAINING INFORMATION PROFESSIONALS TO DEAL WITH NEW TECHNOLOGIES1}

\author{
by Jamshid Boheshti
}

\begin{abstract}
Information professionals are increasingly required to perform technical duties dealing with new technologies. Training them to cope with the extended responsibilities has become a challenging task for the library and information studies programs. A systematic approach to conveying the IT (Information Technology) knowledge base to professionals has been suggested here based on a modified version of an educational taxonomy in the cognitive domain. Two continua are constructed dealing with hardware and software knowledge bases. The software continuum consists of three Levels: Application, Analysis/Synthesis and Evaluation/Creation. The hardware continuum is slightly different: Comprehension, Application and Synthesis/Evaluation. The depth of the knowledge base and competency level increases with each level. The continua may be used as guidelines to design a comprehensive curriculum in the IT area. The outcome of the continua may result in an IT curriculum with eight courses or modules, four of which should be required and four optional.
\end{abstract}

\subsection{Introduction}

For the past decade, schools of library and information studies have been struggling to cope with the rapid advances in information technology. Faculty members have had countless meetings to reshape old courses or design new ones to embrace the new technology. Computer laboratories, which were set up years ago with modest budgets are now perhaps the largest drain on the dwindling resources of schools. It is estimated that by the end of this century, $98 \%$ of all new information will be in digital form. (CRISTAL-ED) To keep pace with these new developments, schools of library and information studies have been forced to put even more resources into teaching IT to prepare information professionals for the job market.

Requirements for this job market, however, are not completely clear. In a Forbes article in 1993, David Churbuck stated: "It would be too much to argue that the jobs of the 152,000 librarians in the U.S. are in jeopardy. But it's fair to say that their jobs will change dramatically over the next two decades, courtesy of the Internet computer networking system." (Churbuck 1993). The market for information professionals may be divided into three broad categories: traditional library and information centres where skills of selection, organization and dissemination of information are mainly utilized; libraries without walls or virtual libraries where diverse groups with different backgrounds such as database managers, marketing specialists, communication specialists and the like are needed; and finally the third market segment where technological specialists such as programmers, telecommunication and system specialists are in demand. (Cronin,Stiffler and Day 1993)

1This article is based on a paper presented at the Special Libraries Association 86th Annual Conference, June 14, 1995, Montreal, Quebec. Canada 


\subsection{Knowledge base}

The virtual libraries segment of the market is attracting more information professionals and is one of the few areas in the field which shows any sign of growth. The knowledge base for these professionals may be divided into two broad categories: basic and additional knowledge. Information personnel are required to know all the traditional principles of selection, organization and dissemination of containers of information such as books, joumals, published reports, etc. They also have to move beyond the boundaries of containers. Paul Saffo, a research fellow at the Institute for the Future, suggests that we are in a state "between two revolutions -- one of print, not quite spent, and another of electronics, not quite underway." (Wahlde and Schiller 1993) In the era of virtual libraries, information professionals have to deal with the actual information, or the content as well as the container.

The basic knowledge base for information professionals, therefore, may consist of concepts of selection, organization and dissemination in relation to both non-digitized and digitized libraries. In the case of the former, the basic knowledge base includes collection development, cataloguing and classification using standard tools such as AACR and LC or Dewey systems and the publication of bibliographies and pathfinders. The knowledge base of information professionals in virtual libraries is built upon their existing skills and includes:

\section{Selection}

- User requirement/need analysis: to determine the user's information needs

- Electronic document delivery: vendors' options to deliver digitized information

- Electronic publishing: scholarly and popular information published digitally and delivered on the Intemet or CD-ROMs

\section{Organization}

- Classification and particularly indexing: digitized information including text, still images, moving images, videos, sound

- Z39 standards: implications for organization of information

- MARC: new standards for handling table of contents and eventually full-text

\section{Dissemination}

- Digitization: conversion of printed documents to digitized format

- Intemet: gopher, home-page on World Wide Web, and other available tools.

The additional knowledge base for the virtual library information professional primarily deals with databases and consists of:

Design

- User requirement/need analysis: (same as above, but in the context of design)

- Information analysis: to determine the nature of information and its format
- Systems analysis: feasibility studies, critical path analysis, resource analysis, cost-benefit analysis,

- Human-Computer Interaction (HCI): interface design for input and output

\section{Production}

- Physical and logical structures: files, records, fields, indexes

- Software: database management systems, multimedia authoring systems

- Coding: programming, conversion

Use

- Logical structures of existing systems: files, records, fields, indexes

- Software: database management systems, multimedia systems

\section{Evaluation}

- User requirement/need analysis: (same as Selection)

- Information analysis: integrity, appropriateness

- HCI: evaluation of the interface

- Database: performance, structure

Additional knowledge base should encompass any type of database regardless of the content and format, including textual, numeric, bibliographic, multimedia, expert systems and virtual reality systems.

\subsection{Information Technology in Library and Information Studies Programs}

The role of information technology in both the basic and additional knowledge bases is evident. Ideally every graduate from a masters program in library and information studies (LIS) should possess all the necessary knowledge and skills to be an effective information professional. Unfortunately, we do not live in an ideal world and ultimately options have to be considered and many compromises have to be made. LIS schools have tried to address many issues regarding training, whether for students in their Masters programs or continuing education courses for professionals. These issues may be summarized as:

1. Currency: How up-to-date should schools be? Should they try to anticipate future developments or should they cautiously lag behind the current tide?

2. Breadth: Should schools try to convey a broad knowledge base about hardware and software currently used in information environments? Or should they focus on one or two prevalent applications?

3. Theory versus practice: Should schools concentrate on general principles and theories, or should they focus on training students in specific technologies?

4. Black box versus glass box: Should schools treat the technology as a black box or a glass box? (Large 1993) 
These questions do not lend themselves to easy answers or solutions. At present, none of the American Library Association accredited LIS programs, nor the British schools (Rowley), have a complete agenda in addressing the issue of training information professionals in the IT area.

Recently, the W. K. Kellogg Foundation donated a large grant to the University of Michigan's School of Information and Library Studies (SILS) to map out a comprehensive curriculum for training future information professionals. In its original proposal to Kellogg, SILS states that there is a need for:

A person with understanding and deep commitment to human users and use of information, whoever and wherever they are. A person with the knowledge and skills to organize and preserve information for intellectual access. A person who can effectively use and shape both current and emerging digital systems technology to serve these ends. (CRISTAL-ED)

The Information and Technology Systems Task Force of the School has proposed a plan for teaching IT, consisting of the following modules.

1. Information Design and Human Needs: demonstrates the importance of effective organization of information and interface design in products and services.

2. *Mosaic Searching and the Internet: Internet Addresses, Gopher, FTP

3. *Usenet Newsgroups: searching for information

4. *Introduction to Electronic Mail, LISTSERVs and Electronic Conferencing

5. A Historical Approach to Information Technology from Hollerith to Graphics: Early machines, computer memory, tubes, transistors, integrated circuits, magnetic storage, cores, disks, tapes, optical storage

6. Digital Technology: Data storage, electronic and electromechanical storage, from characters to graphics, stored programs, conversion of alphanumeric data to binary, ASCII codes, decimal to binary conversions.

7. Computer Architecture (Simplified): Hardware components of the personal computer, types of computers, software; operating systems, compilers and programming languages, application software

8. Database Management Systems, Design and Operation

9. Information Retrieval and Indexing: Boolean searching, recall, precision, and relevance

10. *HTML Authoring: Creating a home page, designing an effective home page

11. Telecommunications: Data Communication and Networking: Telephone systems, wireless, cellular, paging, television and radio
12. Concepts of Systems Design and Analysis: System analysis, feasibility studies, critical path analysis, time-motion studies, resource analysis, cost-benefit/effectiveness analysis, organizational impact, system maintenance

13. Intellectual Foundations of Information Technology: History of science, scientific revolutions and paradigms, determinism since the enlightenment, the computer as the ultimate deterministic machine, the computer as a metaphor for human intelligent behaviour

* refers to modules which may not need formal classroom training and can be taught in non-credit courses in laboratories.

SILS's proposal is a very useful outline, upon which other schools may design their IT curriculum. The outline, however, does not address certain questions raised earlier; theory versus practice, broad knowledge of many systems versus an in-depth comprehension of a few.

Perhaps the most crucial aspect of training information professionals in the IT area is the level of competency, or the depth of the knowledge base. SILS's proposal may be taught in one three-credit course, in which case each module would correspond approximately to one three-hour session. On the other hand, the proposal may be divided into at least seven major three-credit courses, comprising one third to one half of the LIS curriculum.

\subsection{An Educational Continuum}

To analy ze the input and the outcome of a training program a more systematic approach is needed. In his "Stating Objectives for Classroom Instruction," Norman Gronlund uses a taxonomy of educational objectives, which was originally developed by Bloom in the 1950s. (Gronlund 1991) The taxonomy is divided into three domains; cognitive, affective and psychomotor. The cognitive domain is of interest to us as the discussion about other domains relates more to children and is primarily age dependent.

Cognitive domain is categorized in a hierarchical order, from simple to complex behaviour. Each category is assumed to include the behaviour at a lower level. Major categories in the cognitive domain are:

1. Knowledge: remembering of previously leamed material, involves recall of a wide range of information from specific to general, from factual to theories. (e.g.; knows common facts and reference sources)

2. Comprehension: the ability to grasp the meaning of material, includes translating material from one form to another, interpreting material (summarizing), estimating future trends, predicting effects. (e.g.; abstracting)

3. Application: the ability to use leamed material in new situations, includes application of rules, methods, concepts and theories. (e.g.; cataloguing) 
4. Analysis: the ability to break down complex material into its components so that its organizational structure is understood, includes identification of the parts, analysis of the relationships. (e.g.; classification)

5. Synthesis: the ability to put parts together to form a new whole, includes the production of an original document. (e.g.; research proposal)

6. Evaluation: the ability to judge the value of material for a given purpose, including judgments based on definite criteria. (e.g.; evaluation of research reports)

Although this taxonomy was originally designed for elementary and secondary schools, its modified version is used to construct a new taxonomy for the information professional's knowledge base or level of competency in the IT area. The six categories have been divided into three major levels:

Level I: Knowledge/Comprehension/Application. When information professionals reach the end of this level, they are able to understand, interpret and apply IT applications.

Level II: Analysis/Synthesis. By the end of this level information professionals are able to analyze new situations and apply known solutions to problems.

Level III: Evaluation/Creation. At the end of this level, information professionals have the ability to judge and decide on a new course of action to solve a problem or choose the best possible altemative.

These levels are not discrete, but form a continuum, increasing in complexity from Level I to Level III. Each level is built upon the knowledge of the previous level, like a building block. Curriculum in the IT area may be designed by applying this taxonomy to two major domains; software and hardware. Although these domains have been treated separately in the following sections, they are part of the same educational continuum and should be dealt with concurrently. Each domain is dependant on the other and cannot be treated in isolation.

\subsection{The Software Domain}

Figure 1 shows the continuum taxonomy divided into three levels applied to the software domain. A taxonomy should be a general model applicable to many situations, however, the focus of this article is on the microcomputer knowledge base, particularly DOS/Windows environments.

In the upper left-hand comer of Figure 1 is the novice trainee entering the continuum. The first topic in the software domain is a basicknowledge and comprehension of the operating system; its function and operation. As each aspect of the software domain is discussed below, examples of the required knowledge base for the information professional is provided in italics.

\subsection{Level I}

At this level trainees leam about general concepts as well as specific applications such as word processing and spreadsheets. They must be able to apply their knowledge base to new situations, however, the complexity and sophistication level is minimal.

\subsubsection{Operating systems}

The most important software is the operating system, which interprets instructions from the user or an application and translates them for the computer. A fundamental knowledge and comprehension of the operating system is essential for effective use of computers. Operating systems may be divided into two broad categories; command-driven and graphical user interface (GUI) systems. In addition to the following, a knowledge of main frame operating systems, such as MVS, and Job Control Language (JCL) would also be an asset for information professionals.

$\begin{array}{ll}\text { DOS } & \text { internallexternal commands: copy, dir,format, } c d \\ \text { UNIX } & \text { basic commands: } l s, c p, c d \\ \text { Windows, OS } 2 & \begin{array}{l}\text { GUI: desktop, tool bar, menu bar, icons, } \\ \text { buttons, dialogue box }\end{array}\end{array}$

In general, file management skills and knowledge of directories are necessary for effective use of the computer as well as the Intemet.

\subsubsection{Word processing}

Word processing has become a necessary and indispensable tool for any professional. Recent budget cuts and dwindling resources have resulted in loss of secretarial positions in many institutions. Consequently, middle managers and professionals are compelled to rely on their own typing and word processing skills. Many years ago, LIS schools required their students to learn typing to produce card catalogues. Today, students are obliged to use word processing in almost all their assignments and projects.

WordPerfect Menus and commands: formatting, cut \&
Word
$\begin{aligned} & \text { paste, search \& replace, simple macros, tables, } \\ & \text { graphics }\end{aligned}$

\subsubsection{Common applications}

Word processing is just one of the many common application software available for everyday use. Other applications at this level of continuum include spreadsheets, personal database software and presentation programs. The electronic spreadsheet has become an important management tool, used for budgeting and forecasting. Personal database software is utilized for creating personnel files, telephone directories and similar management tools. Presentation programs are becoming more popular and an effective tool for managers to present their ideas to board members and directors of their institutions. 
No Experience

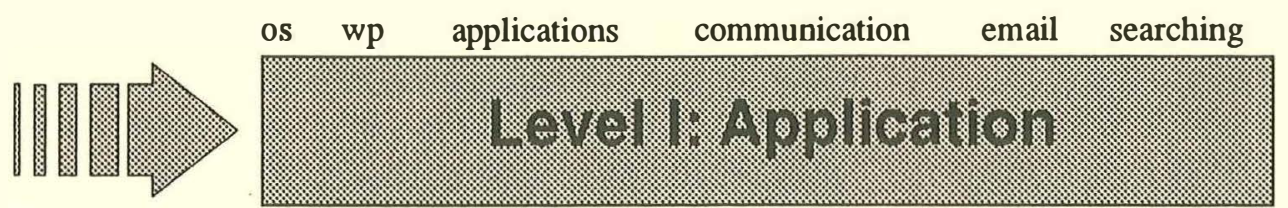
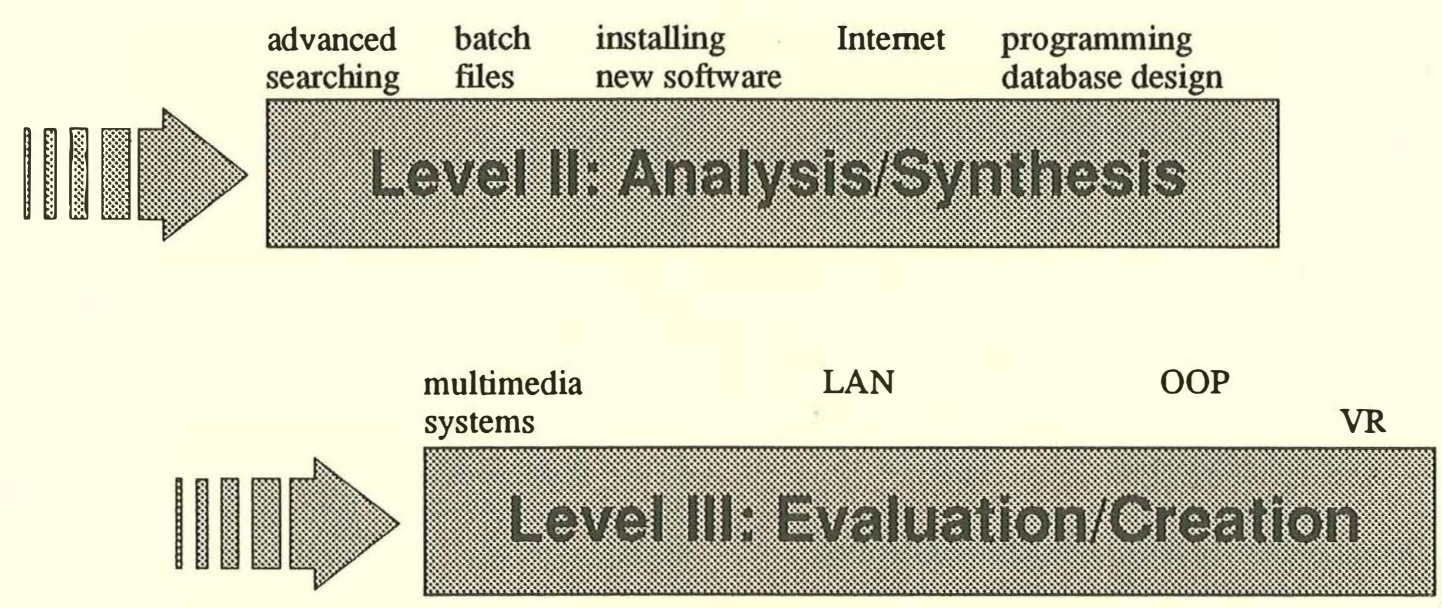

Figure 1. Software Domain Continuum

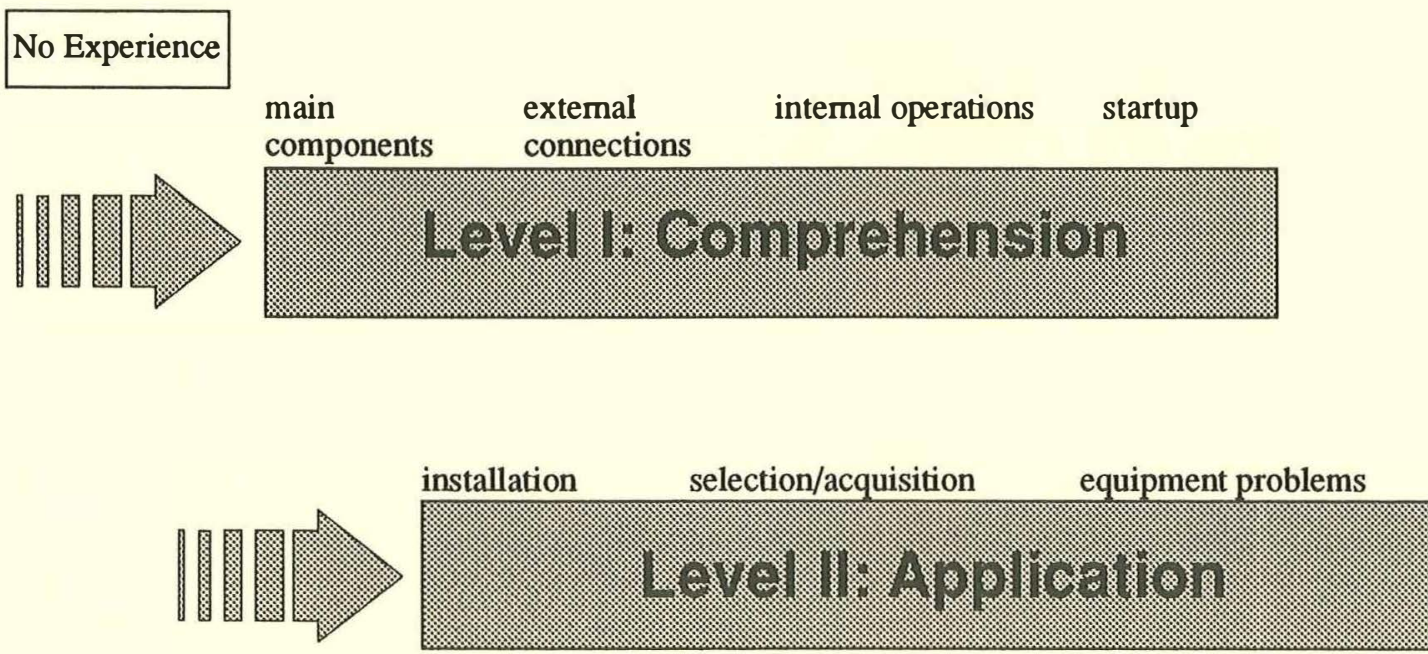

Local Area Network

Wide Area Network

connectivity

\section{Level III: Synthesis/Evaluation}

Figure 2. Hardware Domain Continuum 


\section{1-2-3, Excel}

rows, columns, cells, formulas

FileMaker, Q\&A fields, records, files, attributes, entities

Freelance, PowerPoint charts, graphs, slides

\subsubsection{Communication software}

Since the 1980 s, communication software has played a very important role for information professionals. Uploading online search strategies and downloading the results from systems such as DIALOG have become common techniques in the past decade. Communication software, however, is now more prevalent today, thanks to the Intemet. Unlike some of the other application software, leaming about communication requires a knowledge of protocols, networking, telecommunication and similar issues.

Procomm

Crosstalk

upload, download, protocol, parity, speed

\subsubsection{Email}

Sending electronic messages has become a routine task today, requiring very little technical expertise. Nevertheless, a certain amount of knowledge about the content and the container of the message is required. In general, the trainee should have a basic understanding of networks and data exchange protocols.

\section{QuickMail addresses, protocol, multiplecopies, Microsoft Mail forward, append}

\subsubsection{Shareware and viruses}

Advents in communication have brought shareware, freeware, public domain software, groupware and viruses. It is important to understand the differences among the first three types of software. Shareware programs are made available to public, who must pay a registration fee once they have decided to use the software. Freeware programs are copyrighted by their creators but may be distributed free of charge to other users. Public domain software programs are not copyrighted and are for anyone's use. Thousands of programs which fall into one of these software categories, are currently available through the Internet.

A new type of software called groupware provides tools for professionals to work together. One of the features of groupware is to schedule meeting times by accessing the computerized calendars of all the people involved. Other features include exchanging information and sending reminders for the meetings.

As more shareware and freeware become available, viruses proliferate at an alarming rate. While some are relatively harmless and simply display annoying messages, others are destructive. A basic knowledge of viruses and preventive measures is essential.
F-Prot, Norton

SAM

worm, trojan horse, stealth

\subsubsection{Searching techniques}

Perhaps the most important aspect of training information professionals in the IT area is the online searching techniques. At this level a basic understanding of command language, records, fields, files and indexes is essential. Retrieving information from different databases, including bibliographic and full-text, form an integral part of searching techniques knowledge base.

\section{DIALOG, OPACs keyword searching, Boolean and proximity operators}

\subsection{Level II}

At this level trainees leam how to analyze a problem and apply their knowledge base to find existing solutions. They must move beyond the basic application of software and be able to apply advanced techniques to different situations.

\subsubsection{Advanced searching techniques}

Advanced retrieval techniques are based on the knowledge base gained in Level I and may include different types of databases such as numeric, patent, legal and scientific. It also involves a more in-depth understanding of common command language and the differences and similarities among various systems. Although many CD-ROM multimedia databases have graphical user interfaces (GUI) and may not require the use of common search techniques, information professionals should be thoroughly familiar with their logical file and record structures and comprehend the relationships among various data elements. In addition, they should be able to use hypertext techniques and know its advantages and limitations.

\subsubsection{Software installation and batch files}

Many information professionals need to install new software and CD-ROM applications on their computers at one time or another. Although installation procedures have become much simpler than a few years ago, in many instances professionals are obliged to change systems' configurations. In the DOS and Windows environments, batch files may have to be adjusted or simply rewritten.

Autoexec.bat, reconfiguration, device drivers, memory Config.sys, INI device drivers, memory managers, executable files, paths, commands 


\subsubsection{Standards and the client/server model}

Standards are an integral part of the information world and professionals should be able to comprehend how they are applied in different situations. The client/server model, which has gained much attention recently and is used in many systems, has to rely on standards to function effectively and efficiently.

Data and information exchange $\quad I S O-O S I, Z 39.50, T C P I I P$

\subsubsection{Internet}

Today, many end-users can easily access the Internet by using off-the-shelf software packages which include books, manuals and communication programs. These users are not required to be at Level II of the IT training spectrum to utilize various features of the Net. Information professionals, however, are educators of the Net and need to have an extensive knowledge of its various features and capabilities.

$\begin{array}{ll}\text { Listserv, Usenet } & \begin{array}{c}\text { Read News, selection of listservs, } \\ \text { exchange information } \\ \text { remote logging, OPACs } \\ \text { Telnet }\end{array} \\ \text { FTP } & \begin{array}{c}\text { commary, ASCII, compressed, UNIX } \\ \text { Archie, Gopher, Veronica, Jughead }\end{array} \\ \text { Tools } & \begin{array}{l}\text { hypertext links, multimedia, site selection } \\ \text { WWW }\end{array} \\ \text { GUI Browsers } & \begin{array}{l}\text { Mosaic, Netscape, Air } \\ \text { create homepage }\end{array}\end{array}$

\subsubsection{Programming}

The world's richest man and computer industry's most celebrated personality, Microsoft chairman Bill Gates, recently talked about career paths in a computer camp in Ottawa. His advise to the young adults in the camp was "leam how to program ... leaming a computer language like BASIC is very worthwhile." (The Gazette 1995).

A fundamental knowledge of computer programming is very important for information professionals. Understanding the semantic structures of a language allows the professional to use the computer as a glass box rather than a black box. While the latter approach to computing may be sufficient for paraprofessionals, it is essential for information professionals to take the glass box approach to IT. (Beheshti 1993)

Traditional languages

BASIC, Pascal, C syntactic/semantic structures, sequence, loop, conditional statements, case

\subsubsection{Database design}

While creating flat or two-dimensional tables and files is a relatively simple task, designing a database can be very complex requiring sophisticated techniques and methodologies. Once the database universe, enterprise, entities with their attributes have been identified, data relationships must be established. Data modelling and normalization are integral techniques of relational database design, which may also be used in creating textual, numeric or multimedia databases. Although many database management programs are relatively easy to use, creating a functional database for end-users requires some programming. Information professionals need to construct simple front-ends and interfaces to help end-users retrieve information efficiently and effectively. They need to design templates for paraprofessionals for inputting information into the database. Report generation and mailing lists may also require some programming.

$\begin{aligned} & \text { Relational databases } \\ & \text { dBASE, FoxPro }\end{aligned}$
$\begin{gathered}\text { files, indexes, links, expressions, } \\ \text { programming language } \\ \text { Key tables, join, images, Wizards, Visual } \\ \text { Basic programming language }\end{gathered}$
$\begin{gathered}\text { Bibliographic databases } \\ \text { Inmagic } \\ \text { define fields, indexes, keys; report } \\ \text { generation } \\ \text { Full-text databases }\end{gathered}$
$\begin{aligned} & \text { data entry and word processing modes, } \\ & \text { hypertext links }\end{aligned}$

\subsection{Level III}

At this level trainees learn to evaluate existing options and solutions to a problem, and if required, create new alternatives. They use their knowledge base from Level II to design innovative solutions.

\subsubsection{Mutimedia database design}

Designing multimedia databases may be more complex than relational or bibliographic ones. In addition to traditional data elements, these databases may include still and moving images, sound, animation and hyper-links. Information professionals' knowledge base must be more extensive and in-depth than previous levels to produce cohesive multimedia databases. They should also comprehend the pertinent issues in human-computer interaction (HCI).

Authoring systems

Macromedia Director

still image: gif, tiff files; moving images: MPEG: sound: wavfiles; hyptertext, hypermedia

\subsubsection{LAN}

Networks play a very important role in organizations and they will become even more prevalent as the use of groupware becomes more widespread in the workplace. Setting up a network, however, is not an easy task. Technical as well as organizational skills are required in order to start a local area network. The LAN manager 
has to configure the system, which includes certain amount of programming, create useful interfaces, update and maintain the software. Additional knowledge base at this level also includes familiarity with the client/server architecture.

Network operating system (NOS)

Novell Evaluation, multitasking, multiuser, partitioning, configuration, server, workstations, security, rights, resource allocation

\subsubsection{Object-oriented programming (OOP)}

OOP is a technique that divides a computer program into sets of instructions called objects. The objects are encapsulated because they contain both data and functions that operate on the data. New objects can be created by inheritance, i.e., they "inherit" some properties from existing objects. Objects can also implement the same functions differently (polymorphism). OOP techniques are used to design programs for Windows, where OLE (Object Linking and Embedding) can link data in one program with that created in another program.

Visual Basic encapsulation, inheritance, polymorphism, OLE
C++

\subsubsection{Virtual reality}

Virtual reality (VR) is about simulation of environments. The term is pertinent to any set of stimuli that creates the feeling that one is elsewhere. Different types of VR systems are used in various fields, from medicine and engineering to virtual showrooms. VR systems can be divided into two broad categories: non-immersive systems which do not need expensive hardware and are essentially two-dimensional images projected on a computer monitor, and immersive systems which use headmounted displays (HMD), face masks or multiple large projection screens. Regardless of the techniques used, many current VR applications "do not attempt to fool the user into thinking he or she is really elsewhere but simply provide a window into elsewhere." (Newby 1993) Although VR is still in its infancy, it may have a profound impact on information management. The knowledge and skill required for creating VR applications are based on all the previous Levels discussed above. In addition, information professionals should be familiar with designing and creating expert systems.

\subsection{Hardware domain}

Figure 2 shows the continuum taxonomy divided into three levels applied to the hardware domain. This taxonomy is slightly different from the software domain's taxonomy. The knowledge Levels and the entire continuum have been shifted to accommodate information professionals, since it is assumed that they do not need to have the same level of competencies as in the software domain. While Level I consists of Knowledge/Comprehension, Level II includes Application/Analysis and Level III contains Synthesis/Evaluation. Information professionals are not expected to create new solutions in the hardware domain.

In the upper left-hand comer of Figure 2 is the novice trainee entering the continuum. The first topic in the hardware domain is a basic knowledge and comprehension of the components of a stand-alone microcomputer. As each aspect of the hardware domain is discussed below, examples of the required knowledge base for the information professional is provided in italics.

\subsection{Level I}

At this level trainees learn about general concepts and gain some understanding of computer components and their functions. They must be able to apply their knowledge base to existing situations of minimal complexity.

\subsubsection{Components}

It is imperative for information professionals to know the components of a computer. When they need to select and acquire hardware, they must be able to name the components and have a basic understanding of their functions.

Components $\begin{gathered}\text { motherboard, chips, } C P U, \text { control unit, } A L U, \text { bus, } \\ \text { RAM, DRAM, ROM, ports, hard disk, pixel, } \\ \text { power supply }\end{gathered}$
Peripherals
$\begin{gathered}\text { printers, scanners, video input, lightpens, plotters, } \\ \text { voice synthesizers, monitors, modems, } C D-R O M\end{gathered}$

\subsubsection{Standard external connections}

While Macintosh owners have always enjoyed easy access and ergonomically designed connection ports, until recently other microcomputers owners have not been as fortunate. Professionals should have a basic knowledge of different types of connections and their purposes.

\section{Physical interfaces parallel ports; $D B-25 F$, serial ports; $R S$-232, DB-9, SCSI (Small Computer System Interface), RS-423, MIDI}

\subsubsection{Intemal operations}

Information professionals are not required to be electronic engineers, but they should have a good grasp of the internal operations of a computer. They should understand the relationships among various components.

Operations bits, bytes, machine cycle, memory cache, I-time, E-time, clock speed, address, seek time, conventional memory, upper memory 


\subsubsection{Startup}

Although starting or booting a stand-alone microcomputer seems very simple and a routine task, trainees should have a thorough understanding of the procedures involved. When the computer does not boot properly, for example in case of a weak battery and loss of the configuration information in CMOS, the information professional should be able to explain the problem accurately to a technician.

Boot

$$
\begin{aligned}
& \text { POST (Power On Self Test), BIOS(Basic Input } \\
& \text { Output System), CMOS (Complimentary Metal } \\
& \text { Oxide Semiconductor), warm and cold boots }
\end{aligned}
$$

\subsection{Level II}

At this level trainees learn how to apply their existing knowledge base to solve hardware problems or analyze situations they may encounter. They must move beyond Level I.

\subsubsection{Installation}

Adding new components, such as expansion cards, in a microcomputer have become a routine operation. Although technicians usually install and maintain computer components, in many circumstances professionals have to do these tasks.

Installation sound boards, video boards, multimedia kits, memory boards, hard disks, CD-ROM units

Installation also requires upgrading the CMOS information unless a plug-and-play system is used. CMOS reconfiguration requires an in-depth knowledge of the internal components.

\subsubsection{Selection and acquisition}

Once the fundamental knowledge has been acquired, information professionals are ready to select an appropriate computer for their specific needs. They may choose to purchase a basic machine and add expansion boards and peripherals later, or buy a fully configured system.

Selection memory and storage capacities, speed, hardware and software compatibility, platforms, expendability, obsolescence, warranties and service, cost

\subsubsection{Equipment problems}

Equipment failure does not happen often, but when it does consequences can be grave. A general knowledge of detection and maintenance techniques would save hours of frustration and facilitates intelligent communication with the experts.
Software tools repair and maintain hard disks, unerase files, recover lost files, detect memory problems, detect problems with peripheral devices

\subsection{Level III}

At this level trainees leam to evaluate existing options and solutions to a problem and choose the most appropriate one. They use their knowledge base from Level II for evaluation purposes.

\subsubsection{LAN}

Unlike purchasing a stand-alone computer, setting up a local area network is a complex procedure. The information professional needs to conduct a requirement analysis, plan various scenarios, implement the best plan, maintain and update the solution.

LAN topology: ring, bus; bandwidth, medium:I0 BASET, fiber optic; implementation: Ethernet; multiplexes, hubs, server/workstations

\subsubsection{WAN}

LANs can be attached to wide area networks (WAN) through standard communication protocols. The information professional requires extensive telecommunication skills and broad networking knowledge to design and create a WAN.

WAN LANs, microwave and satellite communication, ISND (Integrated Services Digital Network), FDDI (Packet Fiber Distributed Interface), OSI (Open System Interconnection)

\subsection{IT Curriculum}

Two separate but dependent educational continua have been proposed here to train information professionals for new technologies. Each consists of three Levels corresponding to the depth of the knowledge base or level of competency. The educational continua may be used as guidelines for designing a comprehensive curriculum in the IT area. An example of a curriculum is suggested below.

IT 1: Level I. Modules include computer hardware components and their functions, internal operations, operating systems, startup, word processing, applications, communication software, email, and basic searching techniques.

IT 2: Level II. Modules include advanced searching techniques, installation of hardware components, installation of new software and batch files, selection of new hardware, equipment problems. 
IT 3: Level II. Modules include the Internet.

IT 4: Level II. Modules include computer programming and database design. Many database management software contain languages which may be used to teach programming skills.

IT 5: Level III. Modules include designing multimedia systems.

IT 6: Level III. Modules include planning and implementing LANs, connectivity to other networks, and telecommunication.

IT 7: Level III. Modules include Object-oriented programming

IT 8: Level III. Modules include designing virtual reality systems.

IT 1 may be a credit or non-credit course offered in the first term of a masters program. As more students and professionals gain computer knowledge, this course may be replaced by the more advanced IT 2 course in the near future. IT 2 is an essential course, since in many organizations the responsibility of equipment selection, upgrading and maintenance has been given to information professionals. In addition, they are required to install new soft ware and connect their computers to the Internet. Information professionals are also obliged to conduct workshops and training sessions on the Intemet, including setting up homepages on the World Wide Web. IT 3, which is now becoming an important part of the curriculum in many LIS schools, should include all the necessary information to build a knowledge base for the Internet. IT 4 deals with programming and database designand is a valuable course, which many schools have integrated into their curriculum. IT 5 through IT 8 may be taught as optional courses.

\subsection{Conclusions}

Tom Peters once said: "Thanks to technology, the world is going bonkers. And it's going to get more bonkers - bonkers squared in a few years with bonkers cubed on the way." (interview published in Forbes ASAP, March 29, 1993. Cited in CRISTALED) Technology is moving at a very rapid pace and training information professionals to deal with it is a challenge faced by all educational institutions. Although the W. K. Kellogg Foundation grant to the University of Michigan's SILS to map out a comprehensive curriculum is a first step to meet this challenge, a systematic approach in designing the IT curriculum is needed. In this paper, one method is outlined, however, this should be only the beginning of a series of discussions and consultations for training information professionals to deal with new technologies.

\section{References}

Beheshti, J. (1993) Computer Programming in LIS Education: a Necessity? Education for Information 11(2):123-136.

Churbuck, David C. (1993) Good-bye, Dewey Decimals. Forbes 151:204-5.

CRISTAL-ED. Coalition on Reinventing Information Science, Technology and Library Education. http://unw.sils.umich.edu/ Publications/CRISTALED/KelloggHomePage.html

Cronin, B., Stiffler, M., and Day, D. (1993) The Emergent Market for Information Professionals: Educational Opportunities and Implications. Library Trends 42(2):257-76.

Gronlund, Norman E. (1991) How to Write and Use Instructional Objectives. Toronto: Collier Macmillan.

Large, Andrew. (1993) Information Technology and Education for Library and Information Studies: the Challenge. The Canadian Journal of Information and Library Science 18(1):2333.

"Mr. Gates Gives Advice," Gazette (Montreal), 29 July 1995, sec. D, p.2.

Newby, Gregory B. (1993) Virtual Reality. In Annual Review of Information Science and Technology (ARIST). vol. 28. Edited by Martha E. Williams. Medford, N.J.: Leamed Information.

Rowley, J.E. (1994) Teaching IT Skills to Library and Information Studies Students: Some Reflections. Education for Information 12:235-245.

Wahlde, Barbara von, and Schiller, N. (1993) Creating the Virtual Library: Strategic Issues. In The Virtual Library: Visions and Realities. Edited by Lavema M. Saunders. Westport, CT: Meckler Publishing.

Jamshid Beheshti is Associate Professor at the Graduate School of Library and Information Studies, McGill University, 3459-McTavish Street, Montreal, Quebec, Canada, H3A 1 Y1

voice: (514) 398-4204

fax: (514) 398-7193 